\title{
The neural correlates of intimate partner violence in women
}

\author{
SJ Flegar', JP Fouche', E Jordaan', S Marais'1, B Spottiswoode ${ }^{1}$, DJ Stein², B Vythilingum² \\ 1Department of Psychiatry, Stellenbosch University, South Africa \\ 2Department of Psychiatry and Mental Health, University of Cape Town, South Africa
}

\begin{abstract}
Objective: To examine hippocampal volume and white matter tracts in women with and without intimate partner violence (IPV). Method: Nineteen women with IPV exposure in the last year, and 21 women without IPV exposure in the last year underwent structural magnetic resonance imaging (MRI) including diffusion tensor imaging (DTI) sequences. Additional data on alcohol use and presence of psychiatric disorder was collected. Differences in fractional anisotropy (FA) between the two groups were examined, using a statistical model that included demographic measures, alcohol use and psychiatric disorder. Results: IPV subjects did not demonstrate significantly different hippocampal volumes compared to subjects without recent IPV. FA was, however, significantly reduced in the body of the corpus callosum of IPV subjects. Adjusting for age, alcohol use, smoking and psychiatric diagnosis did not change the significance of the result. Conclusion: Data on hippocampal volume in IPV are inconsistent, perhaps reflecting the fact that multiple factors influence this measure. Reduced FA in the body of the corpus callosum in IPV suggests altered integrity of this white matter tract; additional work is needed to address the underlying mechanisms and clinical correlates of this finding.
\end{abstract}

Key Words: Corpus callosum; Hippocampal volume; Intimate partner violence; Neuroimaging

Received: 03-09-2010

Accepted: 02-1 1-2010

doi: http://dx.doi.org/10.4314/ajpsy.v14i4.9

\section{Introduction}

Intimate partner violence (IPV) is a global public health problem. Population-based surveys from developed countries indicate that 1 in 4 women have experienced IPV in their lifetime, and 1 in 10 are current victims. ${ }^{1-2}$ Similarly, a cross-sectional survey from South Africa found that the lifetime prevalence of women experiencing IPV was $24.6 \%$, and $9.5 \%$ in the last year. ${ }^{3}$ The high prevalence of adverse physical and mental health outcomes related to IPV, is well established in the research literature. ${ }^{4-6}$ In a review of mental health outcomes of women with IPV, victims of partner violence were up to 5 times more likely to develop major depressive episode (MDE), suicidality, posttraumatic stress disorder (PTSD) and substance abuse compared to non victims. ${ }^{7}$

There is growing interest in determining the neurobiological correlates of trauma exposure and its sequelae. A number of neurobiological studies of PTSD in

\section{Correspondence}

Dr SJ Flegar

Department of Psychiatry, Stellenbosch University, Stikland Hospital,

De La Haye Avenue, Private Bag X13, Bellville, 7535, South Africa.

email: flegarsj@gmail.com women have specifically recruited subjects with IPV exposure. ${ }^{8-11}$ In one such study, women exposed to IPV displayed deficits in executive functioning, working memory and visuoconstruction. ${ }^{11}$ In another, women with IPV were found to have smaller supratentorial cranial vault volumes, largely due to reduced mesial temporal grey matter particularly in the parahippoocampal region, and reduced frontal and occipital grey matter regions. ${ }^{10}$ Taken together, these findings suggest that IPV may be associated with deficits in cognitive tasks, which are in turn associated with a range of neural circuitry abnormalities.

It is unclear whether these findings are due to the trauma exposure, or to the sequelae of trauma exposure (eg PTSD, $\mathrm{MDE}$ ). In a series of meta-analyses of hippocampal volume in PTSD, persons exposed to trauma (not exclusively IPV) without PTSD had reduced hippocampal volumes compared to healthy non-trauma exposed controls. In the same series, a meta analysis (12 studies, $\mathrm{N}=379$ ) of persons with trauma exposure and PTSD compared to trauma exposed non-PTSD subjects found a significant reduction in the left hippocampal volume in the PTSD group. ${ }^{12}$ There are few studies that have specifically investigated hippocampal volume in IPV exposed subjects. One such study reported no difference in hippocampal volume in women with IPV and PTSD compared to women with 
IPV but without PTSD. ${ }^{10}$ Similarly subjects with alcohol use or psychiatric disorders other than PTSD have been excluded, despite the close association between IPV exposure, alcoholism and a range of disorders. ${ }^{7}$

Furthermore, there are no studies that have examined white matter tracts in women with IPV. We aimed to study a group of women with recent IPV exposure, which allowed the inclusion of alcohol use and comorbid psychiatric disorders, and aimed to include these as covariants in our statistical model. We focused in particular on hippocampal volume, given the discrepancy in the research literature, and to assess white matter integrity.

\section{Methods}

\section{Subjects}

Forty women were recruited from a rural community north of Cape Town. Subjects had been part of a larger clinical trial investigating the prevention of fetal alcohol spectrum disorder through a series of brief interventions to change drinking behaviour in pregnant women. ${ }^{13}$ We selected this sample on the basis of the high prevalence of alcohol abuse and IPV in that area. In addition, the field worker was familiar with these women through her work in the main study, which made it easier to recruit subjects given the sensitive nature of our study. Inclusion criteria were women aged 16 to 65 years. Subjects were excluded if 1) they were pregnant, 2) MRI was contra-indicated, 3) there was a history of a neurological disorder, including previous head injury, epilepsy, or cerebrovascular disease, or 4) they had current medical illness. Patients with alcohol use or psychiatric disorders were not excluded.

\section{Interview}

All participants were seen by a psychiatrist who completed a standardized assessment. The Abuse Assessment Screen (AAS) was used to classify participants as cases or controls: cases $(n=19)$ were defined as women who reported IPV in the last year (score $>1$ ), and controls $(n=21)$ were defined as women with no reports of violence in the previous year. The Alcohol Use Disorders Identification Test (AUDIT) and the Mini International Neuropsychiatric Interview (MINI) were used to obtain additional data on alcohol use, and to screen for the presence of psychiatric disorders, in particular PTSD and major depression. All participants gave written informed consent to participate in the study, which was approved by the University of Cape Town Research Ethics Committee.

\section{MRI scanning}

Scanning took place at the Cape Universities Brain Imaging Centre (CUBIC) in Tygerberg Hospital. Images were acquired using a 3 Tesla Siemens Allegra MRI scanner. Scans were taken up to 12 months post delivery to allow for any pregnancy related changes to resolve. Tl MPRAGE images were acquired in the sagittal plane with the following parameters: $\mathrm{TR}=2200, \mathrm{TE}=5.2, \mathrm{FOV}=256 \times 256$ and 160 slices with a thickness of $1 \times 1 \times l \mathrm{~mm}$. For the diffusionweighted images (DWI), 3 averages were obtained and each average consisted of 30 diffusion directions with $\mathrm{b}=1000$ $\mathrm{mm} / \mathrm{s} 2,3 \mathrm{~b}=0 \mathrm{~mm} / \mathrm{s} 2$ images, $\mathrm{TR}=8800$ and $\mathrm{TE}=88$. The images were acquired as a mosaic ( 960 x 960 matrix) with 60 slices per volume and a slice thickness of $2.2 \mathrm{~mm}$.

\section{Hippocampal volume measurements}

The FSL software package FIRST (FMRIB's integrated registration and segmentation tool) was used to determine volume and shape differences for the left and right hippocampus. ${ }^{14-15}$ The process is as follows: the $\mathrm{Tl}$ images of each subject are transformed to Montreal Neurological Institute (MNI) 152 space by a 12-degree affine transformation. After the subcortical structures are aligned, a subcortical mask is applied to locate the different subcortical regions. Segmentation of these regions is then performed according to standardized shape models and voxel intensities. The absolute volumes of the individual structures are calculated, taking into account the transformation parameters of the initial registration. For this study, a boundary correction was applied to the hippocampus to determine which voxels along the boundary belong to the structure. A correction with a z-value of 3 was used. The segmentations and registrations were examined for any misregistration. A vertex statistical analysis was performed on the segmented hippocampus for each subject to examine whether there were any significant differences in hippocampus structure and shape between cases and controls. A total of 2 control and 4 IPV subjects were excluded from this analysis because of problematic registration and artefacts in the left hemisphere of the brain. Thus the final analysis was performed on 19 control subjects and 15 IPV cases.

\section{Diffusion tensor imaging}

Diffusion tensor imaging (DTI) is a non-invasive MRI technique that enables the measurement of the diffusion of water in tissue in order to produce white matter (WM) images. Fractional anisotropy (FA) is the quantitative measure of the directionality of diffusion which varies from zero (diffusivity equal in all directions) to one (entirely unidirectional). Lower FA measures are an index of decreased WM integrity.

\section{Post-processing: Tract-based spatial statistics (TBSS) white matter $F A$ analysis}

The whole analysis was performed in the FMRIB's software library (FSL). ${ }^{16}$ For the data analysis, fractional anisotropy (FA) and mean diffusion (MD) maps were created by firstly conducting brain extraction (BET) and then fitting a tensor model to the raw diffusion data using FMRIB's Diffusion Toolbox (FDT). ${ }^{17}$ The subjects FA data were aligned into a common space using the non-linear registration tool FNIRT. ${ }^{18}$ The mean FA image was then created and thinned to create a mean FA skeleton that represents the centres of all white matter (WM) tracts common to the group. Each subject's aligned FA data were then projected onto the skeleton and resulting data fed into voxelwise cross-subject statistics. A two-tailed unpaired t-test with threshold-free cluster enhancement was used for the two groups. Only clusters that have a p-value $<0.05$ corrected for multiple comparisons were considered. Analysis of variance was used to test whether fractional anisotropy (FA) was different for the IPV exposure group. Covariates age, smoking, alcohol and psychiatric diagnosis, that could possibly affect the relationship, were entered into the model. 


\section{Results}

The demographic variables in Table I were measured at entry to the larger study. There was no significant difference in demographic data (age, level of education, employment, relationship status, and urban settlement) between subjects with recent IPV exposure and control subjects.

The group of women who reported IPV in the previous year, scored significantly higher on the AUDIT (p 0.001). Similarly, current psychiatric morbidity was significantly greater in the IPV subjects relative to those women without reports of IPV in the previous year ( $\mathrm{p} 0.003$ ) - see Table II

\section{IPV FIRST hippocampus volume analysis}

A surface FDR correction for multiple comparisons was performed on the vertex maps of the left and right hippocampus. There was no significant difference in volume between IPV subjects and controls ( $p>0.05)$

Fractional anisotropy (FA) was significantly reduced in the body of the corpus callosum in the IPV exposure subjects ( $p$ 0.0003) - see Figure 1

Age, smoking, alcohol and psychiatric diagnosis can affect white matter tracts. For this reason age, smoking status, alcohol use and presence of psychiatric diagnosis were incorporated as covariates in the statistical model, but this did not impact on the main finding - see Table III

\section{Discussion}

This study yielded several findings. First, women with recent IPV use more alcohol and experience more psychiatric morbidity than women without recent IPV exposure. Second, the hippocampal volume in women with IPV is not reduced compared to women without IPV exposure in the last year. Third, a change in the integrity of WM tracts in the body of the corpus callosum is seen in women exposed to interpersonal violence. Fourth, the abnormality in the WM tracts held true even when demographic factors, alcohol use, and psychiatric diagnosis were incorporated into the model.

The adverse mental health sequelae of IPV are well documented in the literature. ${ }^{19-24}$ Similarly, in our study, women with IPV exposure used more alcohol (26.3\% alcohol dependence, $10.5 \%$ alcohol abuse), had a trend towards

\begin{tabular}{|l|l|l|l|}
\hline \multicolumn{2}{|l|}{ Table I: Demographic variables } \\
\hline Variable & $\begin{array}{l}\text { Controls } \\
n=21 \\
\text { Mean or \% }\end{array}$ & $\begin{array}{l}\text { IPV Cases } \\
n=19 \\
\text { Mean or \% }\end{array}$ & P-Value \\
\hline Age & 25.4 & 22.1 & 0.094 \\
Primary school education only & 42.9 & 26.3 & 0.271 \\
Further training after school & 14.3 & 10.5 & 0.719 \\
Employed & 52.4 & 36.8 & 0.323 \\
Live in town & 76.2 & 89.5 & 0.262 \\
In a relationship & 52.4 & 63.2 & 0.490 \\
Pregnant more than once & 52.4 & 52.6 & 0.987 \\
Smoker & 35 & 63.2 & 0.077 \\
\hline
\end{tabular}

\begin{tabular}{|l|l|l|l|}
\hline \multicolumn{4}{|l|}{ Table II: Psychiatric morbidity } \\
\hline Variable & $\begin{array}{l}\text { Controls } \\
n=21 \\
\text { Mean(\%) }\end{array}$ & $\begin{array}{l}\text { IPV Cases } \\
n=19 \\
\text { Mean (\%) }\end{array}$ & P-Value \\
& 2.5 & 11.2 & 0.001 \\
\hline Audit score & 14.3 & 57.9 & 0.003 \\
Number of comorbid diagnoses on MINI & $1(4.8)$ & $6(31.6)$ & \\
MDD & 0 & $1(5.3)$ & \\
Dysthymia & 0 & $1(5.3)$ & \\
PTSD & 0 & $1(5.3)$ & \\
GAD & $1(4.8)$ & 0 & \\
SAD & $2(9.5)$ & 0 & \\
Agoraphobia & 0 & $2(10.5)$ & \\
Alcohol abuse & 0 & $5(26.3)$ & \\
Alcohol Dependence & 0 & $1(5.3)$ & \\
Metamphetamine dependence & 0 &
\end{tabular}

Table III: Impact of co variates

\begin{tabular}{|l|l|l|l|l|l|}
\hline & $\begin{array}{l}\text { Contro/s } \\
n=21 \\
\text { Mean }\end{array}$ & $\begin{array}{l}\text { Controls } \\
\text { SD }\end{array}$ & $\begin{array}{l}\text { Cases } \\
n=19 \\
\text { Mean }\end{array}$ & $\begin{array}{l}\text { Cases } \\
\text { SD }\end{array}$ & P value \\
\hline $\begin{array}{l}\text { FA } \\
\text { Corpus } \\
\text { Callosum }\end{array}$ & 0.68 & 0.07 & 0.59 & 0.08 & 0.0003 \\
\hline
\end{tabular}

Figure 1

Anatomy: Body of the corpus callosum

Hemisphere: Bilateral

MNI coordinates: X 91, Y 130, Z 96

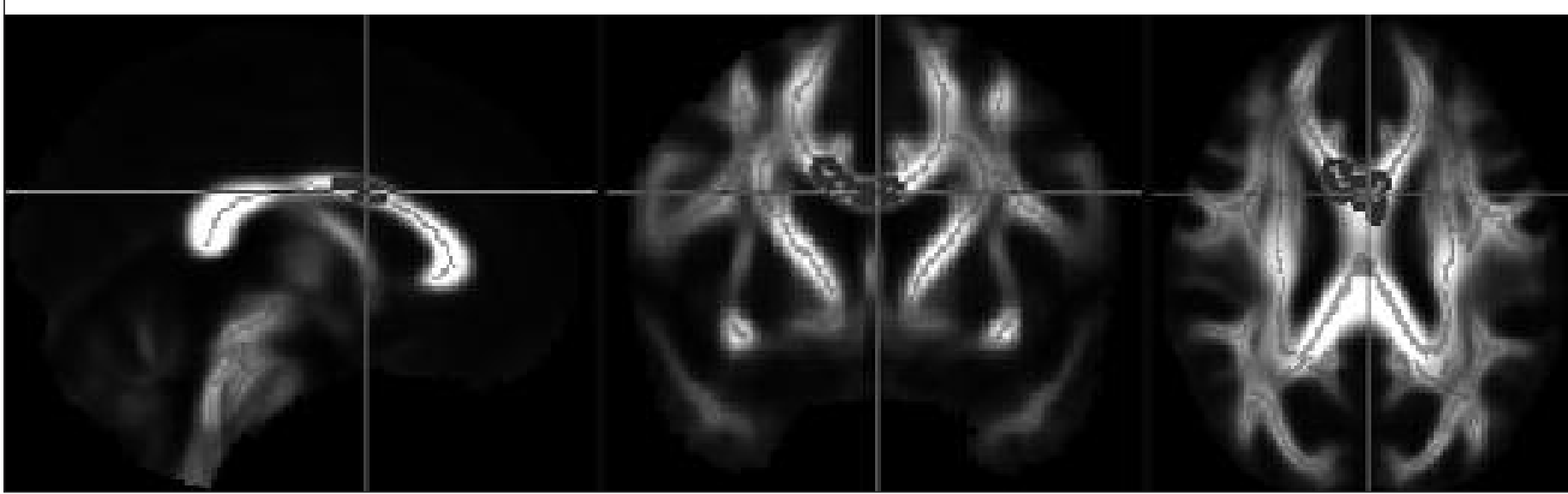


increased nicotine use, and had more psychiatric diagnoses (31.6\% major depression, 15.8\% anxiety disorders) than non-exposed subjects.

Previous literature on hippocampal volume after exposure to trauma has been inconsistent. One the one hand, a series of meta-analyses of structural brain abnormalities in PTSD, ( 6 studies, $N=175$ ) that compared hippocampal volume in persons exposed to trauma without PTSD and healthy controls (HC) reported that the trauma exposed non-PTSD subjects had significantly smaller bilateral hippocampal volumes compared to the HC subjects. ${ }^{25}$ Nevertheless, there is now evidence that in twins with PTSD, deficits in hippocampal functioning precedes exposure to trauma. ${ }^{26}$ Thus it is possible that decreased hippocampal volume is a vulnerability factor for developing PTSD after exposure to trauma, rather than a consequence of exposure to trauma. Indeed, in an adult study of subjects with IPV with and without PTSD, PTSD status did not significantly change the finding that hippocampal volume was not reduced. ${ }^{10}$ Other factors such as age, gender depression, may also influence hippocampal volume and so contribute to inconsistencies in the PTSD literature on hippocampal volume.

The corpus callosum is the largest white matter (WM) fiber bundle in the brain, and connects corresponding areas of cortex between hemispheres. Several studies of paediatric PTSD have reported FA reductions in the corpus callosum. ${ }^{27-28}$ Reduced size of the corpus callosum in male non-human primates exposed to prenatal stress suggests that the mechanisms may involve exposure to stress per se; which may lead to neuronal atrophy. ${ }^{29}$ Further work is needed to explore the clinical correlates and consequences of possible decreased white matter integrity in the corpus callosum, but it is notable that women with IPV do have visuoconstructional deficits. ${ }^{11}$

Women with IPV exposure experience more psychiatric disorders including alcohol abuse, relative to women without IPV exposure, possibly accounting for differences in FA in the corpus callosum. However, alcohol use and psychiatric diagnosis was included as covariate in our analysis, and this did not alter the findings.

Important limitations of the current study are that firstly, the experimental group was not clearly PTSD. The approach taken in this study is to move away from particular diagnoses (such as PTSD) and to compare rather a group of subjects exposed to interpersonal violence, and a group without such exposure (the controls). The former group had more psychiatric diagnoses than the controls, as predicted. However, these diagnoses were somewhat heterogenous, with major depression more common than PTSD. Secondly, relatively few subjects had psychiatric comorbidity. Thus the study was not able to definitively exclude the possibility that subjects with and without psychiatric comorbidity had differential involvement of the hippocampus or white matter. Furthermore, given the relatively small sample size, it is possible that there was insufficient power to detect differences in FA in other WM tracts. FA measurement error is lowest in regions with intrinsically high anisotropy, like the corpus callosum. ${ }^{30}$ Finally, childhood trauma was not assessed in our study, and it possible that this contributed to differences in FA between groups.

\section{Conclusion}

In conclusion, our study found that the corpus callosum was altered in women who reported interpersonal violence, while no difference was seen in the hippocampus. More extensive characterization of such subjects, including longitudinal assessments, could be used to shed light on the clinical significance of these abnormalities and to lay the basis for appropriate intervention strategies.

\section{Acknowledgments}

This study was funded by the Centre for Scientific and Industrial Research (CSIR), and by a fellowship from UCT's Brain-Behaviour Initiative. Special thanks to Sandra Marais, $\mathrm{PhD}$, principal investigator of a larger clinical trial, (from where our participants were drawn), which investigated the efficacy of brief interventions to change drinking behaviour for the prevention of fetal alcohol spectrum disorders. ${ }^{13}$

\section{References}

1. Jones AS, Campbell JC, Schollenberger J, et al. Annual and lifetime prevalence of partner abuse in a sample of female HMO enrollees. Womens Health Issues 1999; 6: 295-305.

2. Ratner PA. The incidence of wife abuse and mental health status in abused wives in Edmonton, Alberta. Can J Public Health 1993; 84: 246-49.

3. Jewkes R, Levin J, Penn-Kakana L. Risk Factors for domestic violence: findings from a South African cross-sectional study. Soc Sci Med 2002;55: 1603-1617.

4. Ludermir A, Schraiber L et al . Violence against women by their intimate partner and common mental disorders. Soc Sci Med 2008; 66: 1008-1018.

5. Coker A, Watkins $K$ et al. Social support reduces the impact of partner violence on health:application of structural equation models. Preven Med 2003;37: 259-267.

6. Dutton $M A$, Green $B$ et al . Intimate partner violence, posttraumatic stress disorder and adverse health outcomes. J Interpersonal Viol 2006; 14:955.

7. Golding, J. M. Intimate partner violence as a risk factor for mental disorders: A meta-analysis. Journal of Family Violence 1999; 14(2): 99-132.

8. Simmons AN, Paulus MP, Thorp SR, Matthews SC, Norman SB, and Stein MB. Functional activation and neural networks in women with posttraumatic stress disorder related to intimate partner violence. Biol Psychiatry 2008; 64: 681-690.

9. Seedat S, Videen JS, Kennedy CM, Stein MB. Single voxel proton magnetic resonance spectroscopy in women with and without intimate partner violence-related posttraumatic stress disorder. Psychiatry Research: Neuroimaging 2005; 139:249- 258.

10. Fennema-Notestine C, Stein MB, Kennedey CM et al. Brain morphometry in female victims of intimate partner violence with and without posttraumatic disorder. Biol Psych 2002; 1: 1089-1101.

11. Stein MB, Kennedy CM, Twamley EW. Neuropsychological function in female victims of intimate partner violence with and without posttraumatic stress disorder. Biological Psychiatry 2002; 52: 1079-1088.

12. Karla A, Schaefer M, Maltad LS, Dorfela D, Rohledera N, Werner A. A meta-analysis of structural brain abnormalities in PTSD. Neuroscience and Biobehavioral Reviews 2006; 30: 1004-1031. 
13. Marais S, Jordaan E, Viljoen D, Olivier L. Modifying alcohol drinking behaviour by means of screening and brief interventions with the aim of preventing Fetal Alcohol Syndrome in a high risk population - a cluster randomized trial. Early Child Development and Care. (In press.)

14. Patenaude B. Bayesian statistical models of shape and appearance for subcortical brain segmentation. D.Phil. Thesis. University of Oxford. 2007

15. Patenaude B, Smith S, Kennedy D, and Jenkinson M. FIRST FMRIB's integrated registration and segmentation tool. In Human Brain Mapping Conference, 2007

16. Smith SM, Jenkinson M, Johansen-Berg $H$, Rueckert D, Nichols TE, Mackay CE, et al. Tract-based spatial statistics: Voxelwise analysis of multi-subject diffusion data. Neurolmage 2006; 31 : 1487-1505

17. Smith SM. Fast robust automated brain extraction. Human Brain Mapping 2002; 17(3): 143-155.

18. Andersson JLR, Jenkinson M, Smith S. Non-linear registration, aka Spatial normalization FMRIB technical report TR07JA2 from Www.fmrib.ox.ac.uk/analysis/techrep

19. Parsons LH, Harper MA. Violent maternal deaths in North Carolina. Obstet Gynecol 1999; 94: 990-93.

20. Counts DA. Female suicide and wife abuse in cross cultural perspective. Suicide Life Threat Behav 1987; 17: 194-204.

21. Bergman B, Brismar B. Suicide attempts by battered wives. Acta Psychiatr Scand 1991; 83: 380-84.

22. Golding JM. Intimate partner violence as a risk factor for mental disorders: a meta-analysis. J Fam Viol 1999; 14: 99-132

23. Diaz-Olavarrieta C, Campbell JC, Garcia de la Cadena C, Paz F, Villa A. Domestic violence against patients with chronic neurologic disorders. Arch Neurol 1999; 56: 681-85

24. Leserman J, Li D, Drossman DA, Hu YJB. Selected symptoms associated with sexual and physical abuse among female patients with gastrointestinal disorders: the impact on subsequent health care visits. Psychol Med 1998; 28: 417-2.5.

25. Karla A, Schaefer M, Maltad LS, Dorfela D, Rohledera N, Werner A. A meta-analysis of structural brain abnormalities in PTSD. Neuroscience and Biobehavioral Reviews 2006; 30: 1004-1031.

26. Bonne O, Brandes D, Gilboa A, Gomori JM, Shenton ME, Pitman RK, Shalev AY. Longitudinal MRI study of hippocampal volume in trauma survivors with PTSD. American Journal of Psychiatry 2001; 158: 1248-1251

27. De Bellis MD, Keshavan MS, Shifflett H, Lyengar S, Beers SR, Hall J, Moritz G. Brain structures in pediatric maltreatmentrelated posttraumatic stress disorder: a sociodemographically matched study. Biological Psychiatry 2002; 52: 1066-1078.

28. De Bellis MD, Keshavan MS, Clark DB, Casey BJ, Giedd JN et al. Developmental traumatology. Part II: brain development. Biological Psychiatry 1999; 45: 1271-1284

29. Coe CL, Lulbach GR, Schneider ML. Prenatal disturbance alters the size of the corpus callosum in young monkeys. Developmental Psychobiology 2002; 41: 178-185.

30. Pierpaoli C, Basser PJ. Toward a quantitative assessment of diffusion anisotropy. Magn Reson Med 1996; 36: 893-906.

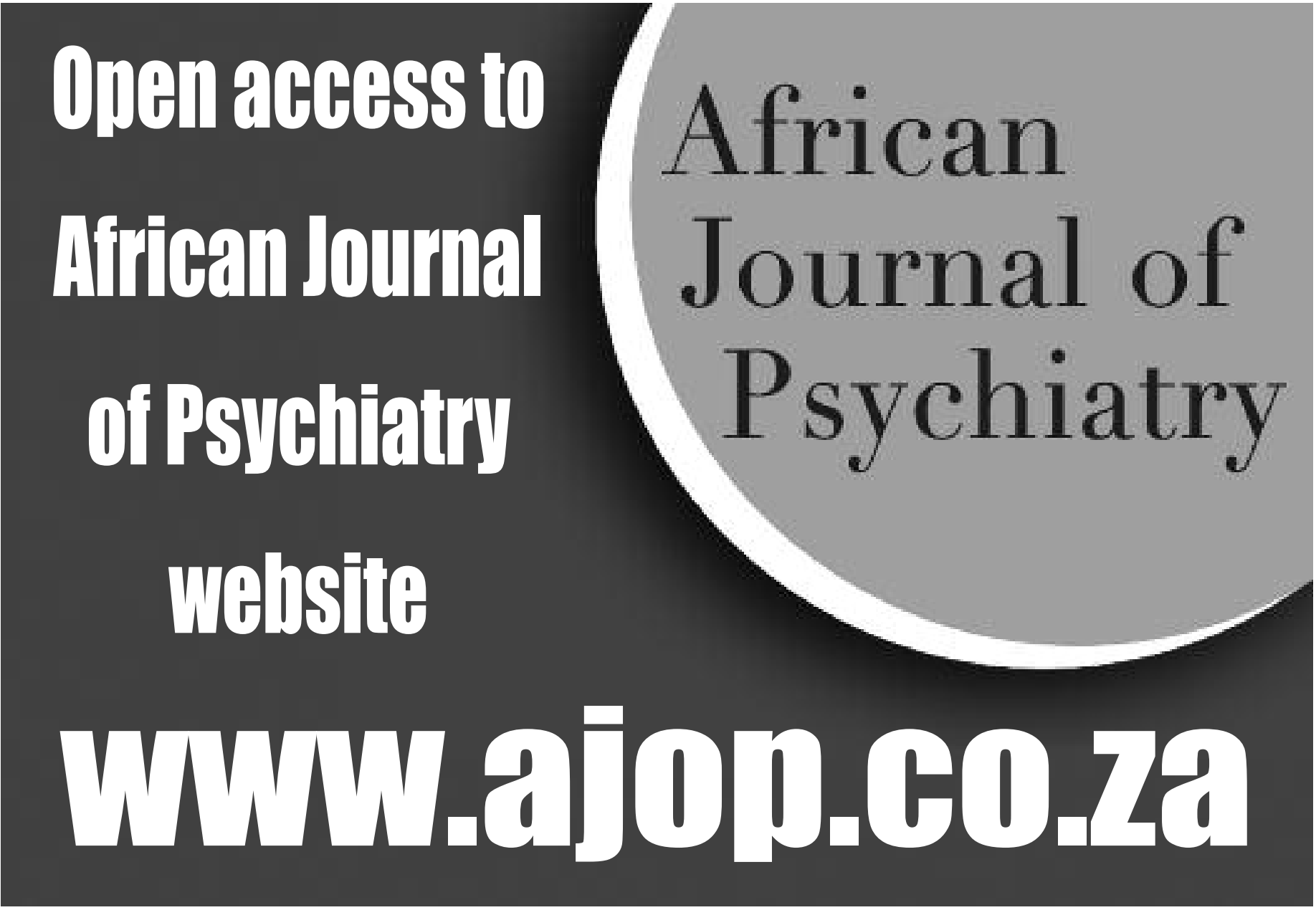

